\title{
When are sequences of Boolean functions tame?*
}

\author{
Malin P. Forsström ${ }^{\dagger}$
}

\begin{abstract}
In [10], Jonasson and Steif conjectured that no non-degenerate sequence of transitive Boolean functions $\left(f_{n}\right)_{n \geqslant 1}$ with $\lim _{n \rightarrow \infty} I\left(f_{n}\right)=\infty$ could be tame (with respect to some $\left(p_{n}\right)_{n \geqslant 1}$ ). In a companion paper [5], the author showed that this conjecture in its full generality is false, by providing a counter-example for the case when, at the same time, $\lim _{n \rightarrow \infty} n p_{n}=\infty$ and $\lim _{n \rightarrow \infty} n^{\alpha} p_{n}=0$ for some $\alpha \in(0,1)$. In this paper we show that with slightly different assumptions, the conclusion of the conjecture holds when the sequence $\left(p_{n}\right)_{n \geqslant 1}$ is bounded away from zero and one.
\end{abstract}

Keywords: Boolean functions.

MSC2020 subject classifications: $60 \mathrm{~K} 99$.

Submitted to ECP on February 22, 2021, final version accepted on November 3, 2021.

Supersedes arXiv : 0000-0003-2555-8521.

\section{Introduction}

The aim of this paper is to show that with slightly different assumptions, a conjecture made by Jonasson and Steif in [10] is true. The results included in this paper complements the earlier paper [5] by the author, which shows that the conjecture, in its full generality, is false.

Before presenting the conjecture and the main result of this paper, we will need some notation and definitions, which we now introduce. For each $n \geqslant 1$, fix some $p_{n} \in(0,1)$ and let $X^{(n)}=\left(X_{t}^{(n)}\right)_{t \geqslant 0}$ be the continuous time $p_{n}$-biased random walk on the $n$-dimensional hypercube $\{0,1\}^{n}$ defined as follows. For each $i \in[n]:=\{1,2, \ldots, n\}$, let $\left(X_{t}^{(n)}(i)\right)_{t \geqslant 0}$ be the continuous time Markov chain on $\{0,1\}$ which at random times, distributed according to a rate one Poisson process, is assigned a new value, chosen according to $\left(1-p_{n}\right) \delta_{0}+p_{n} \delta$, independently of everything else. For each $t \geqslant 0$, we let $X_{t}^{(n)}:=\left(X_{t}^{(n)}(1), \ldots, X_{t}^{(n)}(n)\right)$. The unique stationary distribution of $\left(X_{t}^{(n)}\right)_{t \geqslant 0}$, denoted by $\pi_{n}$, is the measure $\left(\left(1-p_{n}\right) \delta_{0}+p_{n} \delta_{1}\right)^{\otimes n}$ on $\{0,1\}^{n}$. Throughout this paper, we will always assume that $X_{0}^{(n)}$ is chosen with respect to this measure. When $t>0$ is small, the difference between $X_{0}^{(n)}$ and $X_{t}^{(n)}$ is often thought of as noise which describes a small proportion of the bits having been miscounted or corrupted.

A function $f:\{0,1\}^{n} \mapsto\{0,1\}$ will be referred to as a Boolean function. Some classical examples of Boolean functions are the Dictator function, defined by $\operatorname{Dict}_{n}(x):=x(1)$,

\footnotetext{
*Supported by the Knut and Alice Wallenberg Foundation and the European Research Council, Grant Agreement No. 682537.

${ }^{\dagger}$ Department of Mathematics, KTH Royal Institute of Technology, 10044 Stockholm, Sweden.

E-mail: malinpf@kth.se
} 
the Majority function, defined by $\operatorname{Maj}_{n}(x):=\mathbb{1}\left(\sum_{i=1}^{n} x(i) \geqslant n / 2\right)$ and the Parity function, defined by $\operatorname{Parity}_{n}(x):=\mathbb{1}\left(\sum_{i=1}^{n} x(i)\right.$ is even) (see, e.g., [9, 14]). In this paper we will in general be interested in sequences $\left(f_{n}\right)_{n \in \mathcal{N}}$ of Boolean functions, with $f_{n}:\{0,1\}^{n} \rightarrow$ $\{0,1\}$. Since it is sometimes not natural to require that a sequence of Boolean functions is defined for each $n \in \mathbb{N}$, we only require that a sequence of Boolean functions is defined for $n$ in an infinite increasing sub-sequence $\mathcal{N}$ of $\mathbb{N}$. Such sub-sequences of $\mathbb{N}$ will be denoted by $\mathcal{N}=\left\{n_{1}, n_{2}, \ldots\right\}$, where $1 \leqslant n_{1}<n_{2}<\ldots$. To simplify notation, whenever we consider the limit of a sequence $\left(y_{n_{m}}\right)_{m \geqslant 1}$ and the dependency on $\mathcal{N}$ is clear, we will abuse notation and write $\lim _{n \rightarrow \infty} y_{n}$ instead of $\lim _{m \rightarrow \infty} y_{n_{m}}$.

One of the main objectives of [10] was to, given a sequence of Boolean functions $\left(f_{n}\right)_{n \in \mathcal{N}}$, introduce notation which describes possible behaviours of $\left(f_{n}\left(X_{t}^{(n)}\right)\right)_{t \geqslant 0}$ for large $n$. Two of these definitions, which will be relevant for the current paper, are given in the following definition.

Definition 1.1. Let $\left(f_{n}\right)_{n \in \mathcal{N}}, f_{n}:\{0,1\}^{n} \rightarrow\{0,1\}$, be a sequence of Boolean functions. For $n \in \mathcal{N}$, let $C_{f_{n}}$ denote the (random) number of times in $(0,1)$ at which $\left(f_{n}\left(X_{t}^{(n)}\right)\right)_{t \geqslant 0}$ has changed its value, i.e., let

$$
C_{f_{n}}:=\lim _{N \rightarrow \infty} \sum_{i=0}^{N-1} \mathbb{1}\left(f_{n}\left(X_{i / N}^{(n)}\right) \neq f_{n}\left(X_{(i+1) / N}^{(n)}\right)\right) .
$$

Then $\left(f_{n}\right)_{n \in \mathcal{N}}$ is said to be

(i) tame if $\left(C_{f_{n}}\right)_{n \geqslant 1}$ is tight, that is for every $\varepsilon>0$ there is $k \geqslant 1$ and $n_{0} \geqslant 1$ such that

$$
P\left(C_{f_{n}} \geqslant k\right)<\varepsilon \quad \forall n \in \mathcal{N}: n \geqslant n_{0}
$$

(ii) volatile if $C_{f_{n}} \Rightarrow \infty$ in distribution.

In [10], the authors showed that a sequence of Dictator functions is tame and that a sequence of Parity functions is volatile, while a sequence of Majority functions is neither tame nor volatile. More generally, the authors proved that any noise sensitive sequence of Boolean functions is volatile, while any sequence of Boolean functions which is tame is noise stable. As noted in [5] and [8], there are many sequences of functions which are both noise stable and volatile, and hence the opposite does not hold.

We now describe a few additional properties which a Boolean function can have. First, a Boolean function $f:\{0,1\}^{n} \rightarrow\{0,1\}$ is said to be transitive if for all $i, j \in[n]:=$ $\{1,2, \ldots, n\}$ there is a permutation $\sigma$ of $[n]$ which is such that (i) $\sigma(i)=j$, and (ii) for all $x \in\{0,1\}^{n}$, if we define $\sigma(x):=(x(\sigma(k)))_{k \in[n]}$, then $f(x)=f(\sigma(x))$. We say that $f:\{0,1\}^{n} \rightarrow\{0,1\}$ is increasing if for all $x, x^{\prime} \in\{0,1\}^{n}$ such that $x(i) \leqslant x^{\prime}(i)$ for all $i \in[n]$, we have $f(x) \leqslant f\left(x^{\prime}\right)$. A sequence of Boolean functions $\left(f_{n}\right)_{n \in \mathcal{N}}$ is said to be non-degenerate if

$$
0<\liminf _{n \rightarrow \infty} P\left(f_{n}\left(X_{0}^{(n)}\right)=1\right) \leqslant \limsup _{n \rightarrow \infty} P\left(f_{n}\left(X_{0}^{(n)}\right)=1\right)<1 .
$$

Given $x \in\{0,1\}^{n}$ and $i \in[n]$, let $R_{i} x$ denote the random element in $\{0,1\}^{n}$ obtained by resampling the $i$ th bit of $x$ according to $\left(1-p_{n}\right) \delta_{0}+p_{n} \delta_{1}$. If $f:\{0,1\}^{n} \rightarrow\{0,1\}$, we define the influence of the $i$ th bit on $f$, by

$$
I_{i}(f):=P\left(f\left(X_{0}^{(n)}\right) \neq f\left(R_{i} X_{0}^{(n)}\right)\right) .
$$

Note that this definition differs from the definition of influences in, e.g., [11], [12], and [14] by a factor $2 p_{n}\left(1-p_{n}\right)$, but agrees with the analogue definitions given in, 
When are sequences of Boolean functions tame?

e.g., [6] and [10]. If $I_{i}(f)$ is the same for all $i \in[n]:=\{1,2, \ldots, n\}$, then we say that $f$ is regular. The sum of the influences, $I(f):=\sum_{i \in[n]} I_{i}\left(f_{n}\right)$, is called the total influence of $f$.

In [10], the authors show that a sufficient, but not necessary, condition for a nondegenerate sequence $\left(f_{n}\right)_{n \in \mathcal{N}}$ of Boolean functions to be tame is that $\sup _{n} \mathbb{E}\left[C_{f_{n}}\right]<\infty$ (or equivalently, that $\left.\sup _{n} I\left(f_{n}\right)<\infty\right)$. It is natural to ask if this condition is also necessary for some natural subset of the set of all sequences of Boolean functions. This motivated the following conjecture.

Conjecture 1.2 (Conjecture 1.21 in [10]). If $\left(f_{n}\right)_{n \in \mathcal{N}}$ is a non-degenerate sequence of transitive Boolean functions and $\lim _{n \rightarrow \infty} \mathbb{E}\left[C_{f_{n}}\right]=\infty$, then $\left(f_{n}\right)_{n \in \mathcal{N}}$ is not tame.

Remark 1.3. By the second remark after Conjecture 1.21 in [10], there is a tame sequence of Boolean functions which satisfies all assumptions except non-degeneracy in Conjecture 1.2, and hence this assumption is necessary.

In [6], a family of counter-examples to Conjecture 1.2 was given. In detail, these examples provide counter-examples to Conjecture 1.2 exactly when the sequence $\left(p_{n}\right)_{n \geqslant 1}$ is such that $\lim _{n \rightarrow \infty} n p_{n}=\infty$ and $\lim _{n \rightarrow \infty} n p_{n}^{r}=0$ for some $r \geqslant 2$. The assumption that $\lim _{n \rightarrow \infty} n p_{n}=\infty$ guarantees that, as $n \rightarrow \infty$, the expected number of jumps made by $\left(X^{(n)}\right)_{n \in \mathcal{N}}$ in $(0,1)$ tends to infinity. In particular, if $\limsup _{n \rightarrow \infty} n p_{n}<\infty$, then all sequences of Boolean functions will be tame. Consequently, the family of examples given in [6] show that Conjecture 1.2 is false whenever the sequence $\left(p_{n}\right)_{n \geqslant 1}$ tends to zero sufficiently fast, but still slowly enough for there to be interesting behaviour. In contrast to this result, the main objective of this paper is to show that when $\left(p_{n}\right)_{n \geqslant 1}$ is bounded away from zero and one, any non-degenerate sequence of increasing and regular Boolean functions is non-tame, and hence for $\left(p_{n}\right)_{n \geqslant 1}$ in this range, a version of Conjecture 1.2 holds.

Theorem 1.4. If $\left(p_{n}\right)_{n \geqslant 1}$ satisfies $0<\liminf _{n \rightarrow \infty} p_{n} \leqslant \limsup _{n \rightarrow \infty} p_{n}<1$, and $\left(f_{n}\right)_{n \in \mathcal{N}}$ is a non-degenerate sequence of regular and increasing Boolean functions, then $\left(f_{n}\right)_{n \in \mathcal{N}}$ is not tame.

Remark 1.5. If we compare the assumptions on $\left(f_{n}\right)_{n \in \mathcal{N}}$ in Theorem 1.4 with the assumptions on $\left(f_{n}\right)_{n \in \mathcal{N}}$ in Conjecture 1.2, the property of being increasing is added, however, the requirement of transitivity is replaced with the assumption that $f_{n}$ regular for each $n \in \mathcal{N}$.

Remark 1.6. Very interestingly, the family of counter-examples to Conjecture 1.2 given in [6] show that the conclusion of Theorem 1.4 does not hold if the assumption that $0<\liminf _{n \rightarrow \infty} p_{n} \leqslant \limsup _{n \rightarrow \infty}<1$ is replaced by the assumptions that $\liminf _{n \rightarrow \infty} n p_{n}=\infty$ and $\lim \sup _{n \rightarrow \infty} n^{\alpha} p_{n}<\infty$ for some $\alpha \in(0,1)$. This behaviour mirrors similar discrepancies between the two regimes for $\left(p_{n}\right)_{n \geqslant 1}$ which are present also for other results about Boolean functions (see, e.g., [12]). One reason to expect a difference in behaviour between these regimes is that the latter is exactly the regime for which there are non-degenerate sequences of transitive Boolean functions with finite sized witnesses.

Remark 1.7. Using, e.g., Theorem 1 in [2], one shows that any non-degenerate sequence $\left(f_{n}\right)_{n \in \mathcal{N}}$ of regular Boolean functions satisfies $\lim _{n \rightarrow \infty} I\left(f_{n}\right)=\infty$, where $I\left(f_{n}\right)$ is the so-called total influence of $f_{n}$. By Proposition 1.19 in [10], we have $\mathbb{E}\left[C_{f}\right]=I(f)$, and hence the assumptions of Theorem 1.4 guarantee that $\lim _{n \rightarrow \infty} \mathbb{E}\left[C_{f_{n}}\right]=\infty$. We mention that by definition, if $\left(f_{n}\right)_{n \in \mathcal{N}}$ is not tame, then this must hold.

Remark 1.8. The proof of Theorem 1.4 does not really require that $f_{n}$ is regular for each $n \in \mathcal{N}$, but rather that some positive proportion of the influences are of the same order and correspond to a positive proportion to the total influence. 
When are sequences of Boolean functions tame?

The rest of this paper will be organised as follows. In Section 2, we introduce the Fourier-Walsh expansions of Boolean functions, which will be a crucial tool in the proof of Theorem 1.4. In Section 3, we present an expression for $\mathbb{E}\left[C_{f}^{2}\right]$ in terms of the Fourier-Walsh coefficients of $f$. Finally, in Section 4, we give a proof of Theorem 1.4.

\section{Background and notations}

In this section, we will give a brief introduction to the Fourier-Walsh expansion of Boolean functions, and state and prove some results which will be useful to us. For a more thorough introduction to the of Fourier analysis to understand properties of Boolean functions, we refer the reader to [14].

For the rest of this section, fix some $n \geqslant 1$ and assume that $p_{n} \in(0,1)$ is given. To simplify notation, we let $[n]$ denote the set $\{1,2, \ldots, n\}$.

For functions $f, g:\{0,1\}^{n} \rightarrow\{0,1\}$ and $X_{0}^{(n)} \sim \pi_{n}$, we let

$$
\langle f, g\rangle:=\mathbb{E}\left[f\left(X_{0}^{(n)}\right) g\left(X_{0}^{(n)}\right)\right]
$$

Then $\langle\cdot, \cdot\rangle$ is an inner product on the set of real-valued functions with domain $\{0,1\}^{n}$. For $S \subseteq[n]$ and $x \in\{0,1\}^{n}$, define

$$
\chi_{S}(x):=\prod_{i \in S} \frac{x(i)-p_{n}}{\sqrt{p_{n}\left(1-p_{n}\right)}} .
$$

Then $\left\{\chi_{S}\right\}_{S \subseteq[n]}$ is an orthonormal basis for the space of functions $f:\{0,1\}^{n} \rightarrow \mathbb{R}$, using the inner product $\langle\cdot, \cdot\rangle$ (see, e.g., Chapter 8.4 in [14]). In other words, for any $S, T \subseteq[n]$ we have

$$
\left\langle\chi_{S}, \chi_{T}\right\rangle=\mathbb{1}(S=T) \text {. }
$$

Here $\mathbb{1}$ is the indicator function, so that, e.g., $\mathbb{1}(S=T)$ is equal to 1 if $S=T$ and equal to 0 else. Since $\left\{\chi_{S}\right\}_{S \subseteq[n]}$ is finite, any function $f:\{0,1\}^{n} \rightarrow \mathbb{R}$ has a unique decomposition

$$
f(x)=\sum_{S \subseteq[n]} \hat{f}(S) \chi_{S}(x), \quad x \in\{0,1\}^{n}
$$

where $\hat{f}(S)$ is given by $\left\langle f, \chi_{S}\right\rangle$. Moreover, noting that for all $x \in\{0,1\}^{n}$ and all $S, T \subset[n]$ we have

$$
\chi_{S}(x) \chi_{T}(x)=\chi_{S \Delta T}(x) \prod_{i \in S \cap T}\left(1+\frac{1-2 p_{n}}{\sqrt{p_{n}\left(1-p_{n}\right)}} \cdot \chi_{\{i\}}(x)\right)
$$

it follows that for any $S, T, R \subseteq[n]$,

$$
\mathbb{E}\left[\chi_{S}\left(X_{0}^{(n)}\right) \chi_{T}\left(X_{0}^{(n)}\right) \chi_{R}\left(X_{0}^{(n)}\right)\right]=\mathbb{1}(S \Delta T \Delta R=S \cap T \cap R) \cdot\left(\frac{1-2 p_{n}}{\sqrt{p_{n}\left(1-p_{n}\right)}}\right) \cdot
$$

To simplify the rest of the paper, we will abuse notation slightly and sometimes treat $\pi_{n}$ and the functions in $\left\{\chi_{S}\right\}_{S \subseteq[n]}$ as functions with domain $\{0,1\}^{n}$, and sometimes as real-valued vectors in $\mathbb{R}^{\{0,1\}^{n}}$ in the natural way. We let $1:=(1,1, \ldots, 1) \in \mathbb{R}^{\{0,1\}^{n}}$, and note that $\mathbf{1}=\chi_{\emptyset}$. Analogously, we let $\mathbf{0}:=(0,0, \ldots, 0) \in \mathbb{R}^{\{0,1\}^{n}}$.

For $i \in[n], x \in\{0,1\}^{n}$ and $y \in\{0,1\}$, let $x^{i \mapsto y} \in\{0,1\}^{n}$ be defined by

$$
x^{i \mapsto y}(j):=\left\{\begin{array}{ll}
y & \text { if } j=i \\
x(j) & \text { if } j \neq i,
\end{array} \quad j \in[n] .\right.
$$


Using this notation, for each $i \in[n]$ we define the differential operator $D_{i}$ acting on functions $f:\{0,1\}^{n} \rightarrow\{0,1\}$, by

$$
D_{i} f(x):=f\left(x^{i \mapsto 1}\right)-f\left(x^{i \mapsto 0}\right), \quad x \in\{0,1\}^{n} .
$$

In the next lemma, we use the Fourier-Walsh expansion to describe how these differential operators act on Boolean functions.

Lemma 2.1. For any function $f:\{0,1\}^{n} \rightarrow\{0,1\}, i \in[n]$ and $x \in\{0,1\}^{n}$, we have

$$
D_{i} f(x)=\frac{1}{\sqrt{p_{n}\left(1-p_{n}\right)}} \sum_{T \subseteq[n]: i \notin T} \hat{f}(T \cup\{i\}) \chi_{T}(x) .
$$

For a proof of Lemma 2.1, see, e.g., Section 8.4 in [14].

Before closing this section, we mention that it is easy to verify that (see, e.g., [14, Proposition 8.16]),

$$
\operatorname{Var} f_{n}=\sum_{S \subseteq[n]: S \neq \emptyset} \hat{f}_{n}(S)^{2}
$$

and similarly (see, e.g., [14, Proposition 8.23]), that

$$
I\left(f_{n}\right)=\sum_{S \subseteq[n]: S \neq \emptyset}|S| \hat{f}_{n}(S)^{2} .
$$

\section{An expression for $\mathbb{E}\left[C_{f}^{2}\right]$ using the Fourier coefficients}

The main goal of this section is to give a proof of the following proposition, which will be crucial in the proof of Theorem 1.4.

Proposition 3.1. Let $f:\{0,1\}^{n} \rightarrow\{0,1\}$ be increasing. Then

$\mathbb{E}\left[C_{f}^{2}\right]$

$$
\begin{aligned}
& =\mathbb{E}\left[C_{f}\right]+\mathbb{E}\left[C_{f}\right]^{2} \\
& \quad+\sum_{\substack{S \subseteq[n]: \\
S \neq \emptyset}} \frac{e^{-|S|}-(1-|S|)}{|S|^{2}}\left[\left(1-2 p_{n}\right)|S| \hat{f}(S)+2 \sqrt{p_{n}\left(1-p_{n}\right)} \sum_{i \in[n]: i \notin S} \hat{f}(S \cup\{i\})\right]^{2} .
\end{aligned}
$$

In order to give a proof of this result, we will first introduce some additional notation. After this, we state and prove a number of lemmas from which the claim of Proposition 3.1 will follow.

For the rest of this section, assume that $n \geqslant 1$ and $p_{n} \in(0,1)$ is given. For $i \in[n]$ and $x \in\{0,1\}^{n}$, define

$$
\left(x \oplus e_{i}\right)(j):=\left\{\begin{array}{ll}
1-x & \text { if } j=i \\
x(j) & \text { if } j \neq i,
\end{array} \quad j \in[n] .\right.
$$

We now define two matrices which will be useful throughout the rest of this section. Let $Q_{n}$ be the transition matrix of the discrete time Markov chain indexed by the resampling times of $\left(X_{t}^{(n)}\right)_{t \geqslant 0}$, i.e., for $x, y \in\{0,1\}^{n}$ let

$Q_{n}(x, y):= \begin{cases}\frac{1}{n} \cdot \sum_{i=1}^{n}\left(\left(1-p_{n}\right) \mathbb{1}(x(i)=0)+p_{n} \mathbb{1}(x(i)=1)\right) & \text { if } x=y, \\ \frac{1}{n} \cdot\left(p_{n} \mathbb{1}(x(i)=0)+\left(1-p_{n}\right) \mathbb{1}(x(i)=1)\right) & \text { if } y=x \oplus e_{i} \text { for some } i \in[n], \\ 0 & \text { else. }\end{cases}$

Since for any $x \in\{0,1\}^{n}$ and $i \in[n]$ we have $\mathbb{1}(x(i)=1)=x(i)$ and $\mathbb{1}(x(i)=0)=1-x(i)$, the matrix $Q_{n}$ can equivalently be defined by

$$
Q_{n}(x, y):= \begin{cases}\frac{1}{n} \cdot \sum_{i=1}^{n}\left(\left(1-p_{n}\right)(1-x(i))+p_{n} x(i)\right) & \text { if } x=y, \\ \frac{1}{n} \cdot\left(p_{n}(1-x(i))+\left(1-p_{n}\right) x(i)\right) & \text { if } y=x \oplus e_{i} \text { for some } i \in[n] \\ 0 & \text { else. }\end{cases}
$$


When are sequences of Boolean functions tame?

One can easily show that the functions $\left\{\chi_{S}\right\}_{S \subseteq[n]}$ are eigenvectors of $P_{n}$, and that if we let $I_{n}$ denote the $\left|\{0,1\}^{n}\right|$-dimensional identity matrix, we have

$$
\left(Q_{n}-I_{n}\right) \chi_{S}=-\frac{|S|}{n} \chi_{S}
$$

Next, for each function $f:\{0,1\}^{n} \rightarrow\{0,1\}$, we define the matrices $Q_{\partial f}$ and $Q_{f}$ by

$$
Q_{\partial f}(x, y):=Q_{n}(x, y) \cdot \mathbb{1}(f(x) \neq f(y)), \quad x, y \in\{0,1\}^{n}
$$

and

$$
Q_{f}:=Q_{n}-Q_{\partial f}
$$

When $p_{n}=1 / 2$, the function $Q_{\partial f} \mathbf{1}:\{0,1\}^{n} \rightarrow \mathbb{R}$ is exactly equal to the so-called sensitivity of the function $f$, sometimes denoted by $h_{f}$ (see, e.g., [4] and [14]). When $p_{n} \neq 1 / 2$, the function $Q_{\partial f} \mathbf{1}:\{0,1\}^{n} \rightarrow \mathbb{R}$ can be thought of as a weighted analog of this function.

Given $f:\{0,1\}^{n}$ and $i \in[n]$, recall that we have defined $I_{i}(f)$ to be the probability that $f\left(X_{0}^{(n)}\right)$ changes when we re-randomize the $i$ th bit of $X_{0}^{(n)}$. In other words, we have

$$
I_{i}(f)=\mathbb{E}\left[\left(\mathbb{1}\left(X_{0}^{(n)}(i)=0\right) \cdot p_{n}+\mathbb{1}\left(X_{0}^{(n)}(i)=1\right) \cdot\left(1-p_{n}\right)\right)\left(D_{i} f\left(X_{0}^{(n)}\right)\right)^{2}\right] .
$$

Summing over all $i \in[n]$, we obtain

$$
I(f)=\sum_{i \in[n]} \mathbb{E}\left[\left(\mathbb{1}\left(X_{0}^{(n)}(i)=0\right) \cdot p_{n}+\mathbb{1}\left(X_{0}^{(n)}(i)=1\right) \cdot\left(1-p_{n}\right)\right)\left(D_{i} f\left(X_{0}^{(n)}\right)\right)^{2}\right]=\pi_{n}^{T} Q_{\partial f} \mathbf{1} .
$$

We will later be interested in how the matrices $Q_{\partial f}$ and $Q_{f}$ acts on the functions in $\left\{\chi_{S}\right\}_{S \subseteq[n]}$. The first step in this direction is the following result.

Lemma 3.2. Let $f:\{0,1\}^{n} \rightarrow\{0,1\}$ and $S \subseteq[n]$. Then

$$
\pi_{n}^{T} Q_{\partial f} \chi_{S}=\left\langle Q_{\partial f} \mathbf{1}, \chi_{S}\right\rangle \text {. }
$$

Proof. Let $D_{\pi_{n}}$ be the diagonal matrix with $\operatorname{diag} D_{\pi_{n}}=\pi_{n}$. Then, since $X^{(n)}$ is reversible, the matrix $D_{\pi_{n}}^{1 / 2} Q_{n} D_{\pi_{n}}^{-1 / 2}$ is symmetric, and hence it immediately follows that $D_{\pi_{n}}^{1 / 2} Q_{\partial f} D_{\pi_{n}}^{-1 / 2}$ is also symmetric. Using this observation, we obtain

$$
\begin{gathered}
\left\langle Q_{\partial f} \mathbb{1}, \chi_{S}\right\rangle=\left(Q_{\partial f} \mathbb{1}\right)^{T} D_{\pi_{n}} \chi_{S}=\mathbb{1}^{T} Q_{\partial f}^{T} D_{\pi_{n}} \chi_{S}=\mathbb{1}^{T} D_{\pi_{n}}^{1 / 2}\left(D_{\pi_{n}}^{1 / 2} Q_{\partial f} D_{\pi_{n}}^{-1 / 2}\right)^{T} D_{\pi_{n}}^{1 / 2} \chi_{S} \\
=\mathbb{1}^{T} D_{\pi_{n}}^{1 / 2}\left(D_{\pi_{n}}^{1 / 2} Q_{\partial f} D_{\pi_{n}}^{-1 / 2}\right) D_{\pi_{n}}^{1 / 2} \chi_{S}=\mathbb{1}^{T} D_{\pi_{n}} Q_{\partial f} \chi_{S}=\pi_{n}^{T} Q_{\partial f} \chi_{S}
\end{gathered}
$$

as desired.

In the next lemma, we give an expression for $\pi_{n}^{T} Q_{\partial f} \chi_{S}$ in terms of the Fourier coefficients of $f$.

Lemma 3.3. For any increasing function $f:\{0,1\}^{n} \rightarrow\{0,1\}$ and $S \subseteq[n]$, we have

$$
\pi_{n}^{T} Q_{\partial f} \chi_{S}=\frac{1}{n}\left[\left(1-2 p_{n}\right)|S| \hat{f}(S)+2 \sqrt{p_{n}\left(1-p_{n}\right)} \sum_{i \in[n]: i \notin S} \hat{f}(S \cup\{i\})\right] .
$$

Remark 3.4. The proof of Lemma 3.3 is the only part of the proof of our main result that directly requires that each function in the sequence $\left(f_{n}\right)_{n \geqslant 1}$ is increasing. For comparison, one can show that the analogue of (3.4) without this assumption is given by

$$
\pi_{n}^{T} Q_{\partial f} \chi_{S}=\frac{\sqrt{p_{n}\left(1-p_{n}\right)}}{n} \sum_{\substack{T, T^{\prime} \subseteq[n]: \\ T \Delta T^{\prime} \subseteq S \subseteq T \cup T^{\prime}}}\left|T \cap T^{\prime}\right| \hat{f}(T) \hat{f}\left(T^{\prime}\right)\left(\frac{1-2 p_{n}}{\sqrt{p_{n}\left(1-p_{n}\right)}}\right)^{\left|S \cap T \cap T^{\prime}\right|}
$$


When $p \neq 1 / 2$, similar expressions appear naturally since for any $f, g:\{0,1\}^{n} \rightarrow\{0,1\}$ and any $S \subseteq[n]$, we have

$$
\left\langle f g, \chi_{S}\right\rangle=\sum_{\substack{T, T^{\prime} \subseteq[n]: \\ T \Delta T^{\prime} \subseteq S \subseteq T \cup T^{\prime}}} \hat{f}(T) \hat{g}\left(T^{\prime}\right)\left(\frac{1-2 p_{n}}{\sqrt{p_{n}\left(1-p_{n}\right)}}\right)^{\left|S \cap T \cap T^{\prime}\right|} .
$$

The main reason we do not consider this general case is that it does not work as well with the inequalities we will apply later.

Proof of Lemma 3.3. Let $f:\{0,1\}^{n} \rightarrow\{0,1\}$ be increasing and let $S \subseteq[n]$. By Lemma 3.2, we have

$$
\pi^{T} Q_{\partial f} \chi_{S}=\left\langle Q_{\partial f} \mathbf{1}, \chi_{S}\right\rangle .
$$

Fix some $x \in\{0,1\}^{n}$. Since $f$ is increasing we have

$$
n Q_{\partial f} \mathbf{1}(x)=\sum_{i \in[n]}\left(p_{n}(1-x(i))+\left(1-p_{n}\right) x(i)\right) D_{i} f(x) .
$$

Note that for any $i \in[n]$, we have

$$
\begin{aligned}
& (1-x(i)) \cdot p_{n}+x(i) \cdot\left(1-p_{n}\right)=p_{n}\left(1-p_{n}\right)\left(2+\frac{1-2 p_{n}}{\sqrt{p_{n}\left(1-p_{n}\right)}} \cdot \frac{x(i)-p_{n}}{\sqrt{p_{n}\left(1-p_{n}\right)}}\right) \\
& \quad=p_{n}\left(1-p_{n}\right)\left(2+\frac{1-2 p_{n}}{\sqrt{p_{n}\left(1-p_{n}\right)}} \cdot \chi_{\{i\}}(x)\right)
\end{aligned}
$$

and that by Lemma 2.1, we have

$$
D_{i} f(x)=\frac{1}{\sqrt{p_{n}\left(1-p_{n}\right)}} \sum_{T \subseteq[n]: i \notin T} \hat{f}(T \cup\{i\}) \chi_{T}(x) .
$$

Combining these observations with (3.5), we obtain

$$
\begin{aligned}
n Q_{\partial f} \mathbf{1}(x) \cdot \chi_{S}(x) & \\
= & \sum_{i \in[n]}\left(2 \sqrt{p_{n}\left(1-p_{n}\right)}+\left(1-2 p_{n}\right) \cdot \chi_{\{i\}}(x)\right)\left[\sum_{T \subseteq[n]: i \notin T} \hat{f}(T \cup\{i\}) \chi_{T}(x)\right] \chi_{S}(x) \\
= & \sum_{i \in[n]}\left(2 \sqrt{p_{n}\left(1-p_{n}\right)} \sum_{\substack{T \subseteq[n]: \\
i \notin T}} \hat{f}(T \cup\{i\}) \chi_{T}(x)+\left(1-2 p_{n}\right) \cdot \sum_{\substack{T \subseteq[n]: \\
i \in T}} \hat{f}(T) \chi_{T}(x)\right) \chi_{S}(x) .
\end{aligned}
$$

Using (2.1), we thus get

$$
\begin{aligned}
& n \sum_{x \in\{0,1\}^{n}} \pi_{n}(x) Q_{\partial f} \mathbf{1}(x) \chi_{S}(x) \\
& \quad=\sum_{i \in[n]}\left(2 \sqrt{p_{n}\left(1-p_{n}\right)} \hat{f}(S \cup\{i\}) \mathbb{1}(i \notin S)+\left(1-2 p_{n}\right) \hat{f}(S) \mathbb{1}(i \in S)\right) \\
& \quad=2 \sqrt{p_{n}\left(1-p_{n}\right)} \sum_{i \in[n]: i \notin S} \hat{f}(S \cup\{i\})+\left(1-2 p_{n}\right)|S| \hat{f}(S) .
\end{aligned}
$$

This concludes the proof.

In the next lemma we, given a Boolean function $f:\{0,1\}^{n} \rightarrow\{0,1\}$, express the moment generating function of $C_{f}$ as a sum whose terms depend on the matrices $Q_{n}$ and $Q_{\partial f}$. 
When are sequences of Boolean functions tame?

Lemma 3.5. Let $f:\{0,1\}^{n} \rightarrow\{0,1\}$. Then the moment generating function of $C_{f}$ is given by

$$
\mathbb{E}\left[e^{s C_{f}}\right]=\sum_{k=0}^{\infty} \frac{n^{k}}{k !} \pi_{n}^{T}\left(\left(Q_{n}-I_{n}\right)+\left(e^{s}-1\right) Q_{\partial f}\right)^{k} \mathbf{1}, \quad s \in \mathbb{R} .
$$

Proof. Let $f:\{0,1\}^{n} \rightarrow\{0,1\}$ and let $T_{n}$ be the (random) number of times in $(0,1)$ when bits are resampled. By definition, $T_{n}$ has a Poisson distribution with rate $n$. This implies in particular that

$$
\mathbb{E}\left[e^{s C_{f}}\right]=\mathbb{E}\left[\mathbb{E}\left[e^{s C_{f}} \mid T_{n}\right]\right]=\sum_{k=0}^{\infty} \frac{e^{-n} n^{k}}{k !} \mathbb{E}\left[e^{s C_{f}} \mid T_{n}=k\right] .
$$

We now express $\mathbb{E}\left[e^{s C_{f}} \mid T_{n}=k\right]$ in terms of the matrices $Q_{f}$ and $Q_{\partial f}$. To this end, note first that each update of $X^{(n)}$ corresponds to an entry of $Q_{n}$. Moreover, we have $Q_{n}=Q_{f}+Q_{\partial f}$, and for each $x, y \in\{0,1\}^{n}$, we have either $Q_{n}(x, y)=Q_{f}(x, y)$ and $Q_{\partial f}(x, y)=0$, or $Q_{n}(x, y)=Q_{\partial f}(x, y)$ and $Q_{f}(x, y)=0$. Finally, note that if $X^{(n)}$ jumps from $x$ to $y$ and $Q_{f}(x, y) \neq 0$, then we will get a contribution of 1 to $e^{s C_{f}}$, while if $Q_{\partial f}(x, y) \neq 0$ then we get a contribution of $e^{s}$ to $e^{s C_{f}}$. Using these observations, we find that

$$
\begin{aligned}
& \mathbb{E}\left[e^{s C_{f}} \mid T_{n}=k\right]=\pi_{n}^{T}\left(Q_{f}+e^{s} Q_{\partial f}\right)^{k} \mathbf{1}=\pi_{n}^{T}\left(\left(Q_{f}+Q_{\partial f}\right)+\left(e^{s}-1\right) Q_{\partial f}\right)^{k} \mathbf{1} \\
& =\pi_{n}^{T}\left(Q_{n}+\left(e^{s}-1\right) Q_{\partial f}\right)^{k} \mathbf{1} .
\end{aligned}
$$

Combining (3.6) and (3.7), we obtain

$$
\mathbb{E}\left[e^{s C_{f}}\right]=\pi_{n}^{T} \sum_{k=0}^{\infty} \frac{e^{-n} n^{k}}{k !}\left(Q_{n}+\left(e^{s}-1\right) Q_{\partial f}\right)^{k} \mathbf{1}=\sum_{k=0}^{\infty} \frac{n^{k}}{k !} \pi_{n}^{T}\left(\left(Q_{n}-I_{n}\right)+\left(e^{s}-1\right) Q_{\partial f}\right)^{k} \mathbf{1} .
$$

This concludes the proof.

In the next lemma, we use the moment generating function of $C_{f}$ given in Lemma 3.5 to give expressions for the first and second moment of $C_{f}$.

Lemma 3.6. Let $f:\{0,1\}^{n} \rightarrow\{0,1\}$. Then

$$
\mathbb{E}\left[C_{f}\right]=n \cdot \pi_{n}^{T} Q_{\partial f} \mathbf{1}
$$

and

$$
\mathbb{E}\left[C_{f}^{2}\right]=\mathbb{E}\left[C_{f}\right]+2 \sum_{k=2}^{\infty} \frac{n^{k}}{k !} \pi_{n}^{T} Q_{\partial f}\left(Q_{n}-I_{n}\right)^{k-2} Q_{\partial f} \mathbf{1} .
$$

Proof. By Lemma 3.5, for any $s \in \mathbb{R}$, we have

$$
\mathbb{E}\left[e^{s C_{f}}\right]=\sum_{k=0}^{\infty} \frac{n^{k}}{k !} \pi_{n}^{T}\left(\left(Q_{n}-I_{n}\right)+\left(e^{s}-1\right) Q_{\partial f}\right)^{k} \mathbf{1}
$$

Differentiating with respect to $s$ and using that $\pi_{n}^{T}\left(Q_{n}-I_{n}\right)=\left(Q_{n}-I_{n}\right) \mathbf{1}=\mathbf{0}$, we get

$$
\mathbb{E}\left[C_{f}\right]=\left[\frac{d}{d s} e^{s C_{f}}\right]_{s=0}=\left[\frac{d}{d s} \sum_{k=0}^{\infty} \frac{n^{k}}{k !} \pi_{n}^{T}\left(\left(Q_{n}-I_{n}\right)+\left(e^{s}-1\right) Q_{\partial f}\right)^{k} \mathbf{1}\right]_{s=0}=n \cdot \pi_{n}^{T} Q_{\partial f} \mathbf{1}
$$

and

$$
\begin{gathered}
\mathbb{E}\left[C_{f}^{2}\right]=\left[\frac{d^{2}}{d s^{2}} e^{s C_{f}}\right]_{s=0}=\left[\frac{d^{2}}{d s^{2}} \sum_{k=0}^{\infty} \frac{n^{k}}{k !} \pi_{n}^{T}\left(\left(Q_{n}-I_{n}\right)+\left(e^{s}-1\right) Q_{\partial f}\right)^{k} \mathbf{1}\right]_{s=0} \\
=n \cdot \pi_{n}^{T} Q_{\partial f} \mathbf{1}+2 \sum_{k=2}^{\infty} \frac{n^{k}}{k !} \pi_{n}^{T} Q_{\partial f}\left(Q_{n}-I_{n}\right)^{k-2} Q_{\partial f} \mathbf{1}
\end{gathered}
$$

which is the desired conclusion. 
In the next lemma, we expand the terms in (3.9) to get a simpler expression for the second moment of $C_{f}$.

Lemma 3.7. If $f:\{0,1\}^{n} \rightarrow\{0,1\}$, then

$$
\mathbb{E}\left[C_{f}^{2}\right]=\mathbb{E}\left[C_{f}\right]+\mathbb{E}\left[C_{f}\right]^{2}+2 \sum_{S \subseteq[n]: S \neq \emptyset} \frac{e^{-|S|}-(1-|S|)}{|S|^{2}}\left(n \cdot \pi_{n}^{T} Q_{\partial f} \chi_{S}\right)^{2} .
$$

Proof. Let $f:\{0,1\}^{n} \rightarrow\{0,1\}$. Since

$$
Q_{\partial f} \mathbf{1}(x)=\sum_{S \subseteq[n]}\left\langle Q_{\partial f} \mathbf{1}, \chi_{S}\right\rangle \chi_{S}
$$

it follows from Lemma 3.2 that

$$
Q_{\partial f} \mathbf{1}(x)=\sum_{S \subseteq[n]}\left(\pi_{n}^{T} Q_{\partial f} \chi_{S}\right) \chi_{S}
$$

At the same time, for any $S \subseteq[n]$, by (3.1), we have

$$
\pi_{n}^{T} Q_{\partial f}\left(Q_{n}-I_{n}\right)^{k-2} \chi_{S}=\pi_{n}^{T} Q_{\partial f}\left(\left(Q_{n}-I_{n}\right)^{k-2} \chi_{S}\right)=\left(\frac{-|S|}{n}\right)^{k-2} \pi_{n}^{T} Q_{\partial f} \chi_{S}
$$

Combining these observations, we obtain

$$
\begin{gathered}
\pi_{n}^{T} Q_{\partial f}\left(Q_{n}-I_{n}\right)^{k-2} Q_{\partial f} \mathbf{1}=\pi_{n}^{T} Q_{\partial f}\left(Q_{n}-I_{n}\right)^{k-2} \sum_{S \subseteq[n]}\left(\pi_{n}^{T} P_{\partial f} \chi_{S}\right) \chi_{S} \\
=\sum_{S \subseteq[n]}\left(\pi_{n}^{T} P_{\partial f} \mathbf{1}\right) \pi_{n}^{T} Q_{\partial f}\left(Q_{n}-I_{n}\right)^{k-2} \chi_{S}=\sum_{S \subseteq[n]}\left(\pi_{n}^{T} P_{\partial f} \chi_{S}\right)^{2}\left(\frac{-|S|}{n}\right)^{k-2} \\
=\frac{1}{n^{2}} \sum_{S \subseteq[n]}\left(\frac{-|S|}{n}\right)^{k-2}\left(n \cdot \pi^{T} Q_{\partial f} \chi_{S}\right)^{2}
\end{gathered}
$$

Using Lemma 3.6, it immediately follows that

$$
\begin{aligned}
\mathbb{E}\left[C_{f}^{2}\right] & =\mathbb{E}\left[C_{f}\right]+2 \sum_{k=2}^{\infty} \frac{n^{k}}{k !} \cdot \frac{1}{n^{2}} \sum_{S \subseteq[n]}\left(\frac{-|S|}{n}\right)^{k-2}\left(n \cdot \pi^{T} Q_{\partial f} \chi_{S}\right)^{2} \\
& =\mathbb{E}\left[C_{f}\right]+\left(n \cdot \pi^{T} Q_{\partial f} \chi_{\emptyset}\right)^{2}+2 \sum_{S \subseteq[n]: S \neq \emptyset}\left(n \cdot \pi^{T} Q_{\partial f} \chi_{S}\right)^{2} \cdot \frac{1}{|S|^{2}} \sum_{k=2}^{\infty} \frac{n^{k}}{k !}\left(\frac{-|S|}{n}\right)^{k} \\
& =\mathbb{E}\left[C_{f}\right]+\left(n \cdot \pi^{T} Q_{\partial f} \chi_{\emptyset}\right)^{2}+2 \sum_{S \subseteq[n]: S \neq \emptyset}\left(n \cdot \pi_{n}^{T} Q_{\partial f} \chi_{S}\right)^{2} \cdot \frac{e^{-|S|}-(1-|S|)}{|S|^{2}} .
\end{aligned}
$$

Recalling that by Lemma 3.6 we have

$$
\mathbb{E}\left[C_{f}\right]=n \cdot \pi^{T} Q_{\partial f} \mathbf{1}=n \cdot \pi^{T} Q_{\partial f} \chi_{\emptyset},
$$

the desired conclusion follow.

We now combine the lemmas in this section to give a proof of Proposition 3.1.

Proof of Proposition 3.1. By Lemma 3.3, for any $S \subseteq[n]$ we have

$$
\pi_{n}^{T} Q_{\partial f} \chi_{S}=\frac{1}{n}\left[\left(1-2 p_{n}\right)|S| \hat{f}(S)+2 \sqrt{p_{n}\left(1-p_{n}\right)} \sum_{i \notin S} \hat{f}(S \cup\{i\})\right] .
$$


Combining this with Lemma 3.7, we obtain

$$
\begin{aligned}
\mathbb{E}\left[C_{f}^{2}\right]= & \mathbb{E}\left[C_{f}\right]+\mathbb{E}\left[C_{f}\right]^{2}+2 \sum_{\substack{S \subseteq[n]: \\
S \neq \emptyset}} \frac{e^{-|S|}-(1-|S|)}{|S|^{2}}\left(n \pi_{n}^{T} Q_{\partial f} \chi_{S}\right)^{2} \\
= & \mathbb{E}\left[C_{f}\right]+\mathbb{E}\left[C_{f}\right]^{2} \\
& +\sum_{\substack{S \subseteq[n]: \\
S \neq \emptyset}} \frac{e^{-|S|}-(1-|S|)}{|S|^{2}}\left[\left(1-2 p_{n}\right)|S| \hat{f}(S)+2 \sqrt{p_{n}\left(1-p_{n}\right)} \sum_{i \in[n]: i \notin S} \hat{f}(S \cup\{i\}),\right]^{2}
\end{aligned}
$$

which is the desired conclusion.

\section{Proof of the main result}

In this section, we give a proof of Theorem 1.4. The proof of this result will be divided into two lemmas, which we now state and prove.

Lemma 4.1. Let $\left(f_{n}\right)_{n \in \mathcal{N}}$ be a sequence of increasing Boolean functions such that $\lim _{n \rightarrow \infty} \mathbb{E}\left[C_{f_{n}}\right]=\infty$, and assume that there is a constant $C>0$ such that for all $n \in \mathcal{N}$ we have

$$
p_{n}\left(1-p_{n}\right) n \operatorname{Var}\left(f_{n}\right) \leqslant C I\left(f_{n}\right)^{2} .
$$

Then $\left(f_{n}\right)_{n \in \mathcal{N}}$ is not tame.

Remark 4.2. By, e.g., Lemma 6.1 in [7], any increasing Boolean function $f_{n}:\{0,1\}^{n} \rightarrow$ $\{0,1\}$ satisfies $I(f) \leqslant \sqrt{n p_{n}}$. Consequently, by Lemma 4.1, any sequence of increasing Boolean functions which is close to maximizing the total influence must be non-tame.

If $\left(f_{n}\right)_{n \in \mathcal{N}}$ is a sequence of regular and increasing Boolean functions with $p_{n}=1 / 2$ and $\sum I_{i}\left(f_{n}\right)^{2}>c>0$ for all $n \in \mathcal{N}$, then, as observed in the proof of Theorem 1.7 in [1], Theorem 1.1 in [16] immediately implies that the functions $f_{n}$ have uniformly positive correlations with the Majority function. See also Proposition 12.45 and Theorem 12.51 in [9].

Proof of Lemma 4.1. By the Paley-Zygmund inequality, for any $\theta \in(0,1)$ we have

$$
P\left(C_{f_{n}}>\theta \mathbb{E}\left[C_{f_{n}}\right]\right) \geqslant(1-\theta) \frac{\mathbb{E}\left[C_{f_{n}}\right]^{2}}{\mathbb{E}\left[C_{f_{n}}^{2}\right]} .
$$

Since $\lim _{n \rightarrow \infty} \mathbb{E}\left[C_{f_{n}}\right]=\infty$, it immediately follows that if there is a constant $C^{\prime}>0$ such that

$$
\mathbb{E}\left[C_{f_{n}}^{2}\right] \leqslant C^{\prime} \mathbb{E}\left[C_{f_{n}}\right]^{2}
$$

for all sufficiently large $n \in \mathcal{N}$, then $\left(f_{n}\right)_{n \in \mathcal{N}}$ is not tame. Consequently, if we can show that (4.2) holds, then the desired conclusion will follow. To this end, fix some $n \in \mathcal{N}$, and note that by Proposition 3.1, since $f_{n}$ is increasing, we have

$$
\begin{aligned}
\mathbb{E}\left[C_{f_{n}}^{2}\right]=\mathbb{E}\left[C_{f_{n}}\right]+ & \mathbb{E}\left[C_{f_{n}}\right]^{2}+2 \sum_{S \subseteq[n]: S \neq \emptyset} \frac{e^{-S}-(1-|S|)}{|S|^{2}} \\
\cdot & {\left[\left(1-2 p_{n}\right)|S| \hat{f}_{n}(S)+2 \sqrt{p_{n}\left(1-p_{n}\right)} \sum_{i \notin S} \hat{f}_{n}(S \cup\{i\})\right]^{2} . }
\end{aligned}
$$

Since $e^{-|S|} \leqslant 1$ for all $S \subseteq[n]$, this implies that

$$
\begin{aligned}
\mathbb{E}\left[C_{f_{n}}^{2}\right] \leqslant & \mathbb{E}\left[C_{f_{n}}\right]+\mathbb{E}\left[C_{f_{n}}\right]^{2} \\
& +2 \sum_{S \subseteq[n]: S \neq \emptyset} \frac{1}{|S|}\left[\left(1-2 p_{n}\right)|S| \hat{f}_{n}(S)+2 \sqrt{p_{n}\left(1-p_{n}\right)} \sum_{i \notin S} \hat{f}_{n}(S \cup\{i\})\right]^{2} .
\end{aligned}
$$


If we apply the inequality $(a+b)^{2} \leqslant 2\left(a^{2}+b^{2}\right)$, we see that

$$
\begin{aligned}
\mathbb{E}\left[C_{f_{n}}^{2}\right] \leqslant & \mathbb{E}\left[C_{f_{n}}\right]+\mathbb{E}\left[C_{f_{n}}\right]^{2}+4\left(1-2 p_{n}\right)^{2} \sum_{S \subseteq[n]: S \neq \emptyset}|S| \hat{f}_{n}(S)^{2} \\
& +16 p_{n}\left(1-p_{n}\right) \sum_{S \subseteq[n]: S \neq \emptyset} \frac{1}{|S|}\left(\sum_{i \in[n]: i \notin S} \hat{f}_{n}(S \cup\{i\})\right)^{2} .
\end{aligned}
$$

By the Cauchy-Schwarz inequality, we have

$$
\begin{gathered}
\sum_{S \subseteq[n]: S \neq \emptyset} \frac{1}{|S|}\left(\sum_{i \in[n]: i \notin S} \hat{f}_{n}(S \cup\{i\})\right)^{2} \leqslant \sum_{S \subseteq[n]: S \neq \emptyset} \frac{n-|S|}{|S|} \sum_{i \in[n]: i \notin S} \hat{f}_{n}(S \cup\{i\})^{2} \\
=\sum_{T \subseteq[n]:|T| \geqslant 2} \hat{f}(T)^{2} \cdot \frac{|T|(n-(|T|-1))}{|T|-1} \leqslant \sum_{T \subseteq[n]:|T| \geqslant 2} \hat{f}(T)^{2} \cdot 2 n .
\end{gathered}
$$

Now note that

$$
\sum_{S \subseteq[n]: S \neq \emptyset}|S| \hat{f}_{n}(S)^{2}=I\left(f_{n}\right)=\mathbb{E}\left[C_{n}\right]
$$

and

$$
\sum_{S \subseteq[n]:|S| \geqslant 2} \hat{f}_{n}(S)^{2} \leqslant \sum_{S \subseteq[n]: S \neq \emptyset} \hat{f}_{n}(S)^{2}=\operatorname{Var}\left(f_{n}\right) .
$$

Combining (4.3) and (4.4), we thus obtain

$$
\mathbb{E}\left[C_{f_{n}}^{2}\right] \leqslant \mathbb{E}\left[C_{f_{n}}\right]+\mathbb{E}\left[C_{f_{n}}\right]^{2}+4\left(1-2 p_{n}\right)^{2} \mathbb{E}\left[C_{f_{n}}\right]+32 p_{n}\left(1-p_{n}\right) n \operatorname{Var}\left(f_{n}\right) .
$$

Using (4.1) and recalling that $\mathbb{E}\left[C_{f_{n}}\right]=I\left(f_{n}\right)$, we thus obtain

$$
\mathbb{E}\left[C_{f_{n}}^{2}\right] \leqslant \mathbb{E}\left[C_{f_{n}}\right]+\mathbb{E}\left[C_{f_{n}}\right]^{2}+4\left(1-2 p_{n}\right)^{2} \mathbb{E}\left[C_{f_{n}}\right]+32 C \mathbb{E}\left[C_{f_{n}}\right]^{2} .
$$

Since $\lim _{n \rightarrow \infty} \mathbb{E}\left[C_{n}\right]=\infty$, we have $\mathbb{E}\left[C_{n}\right] \leqslant \mathbb{E}\left[C_{n}\right]^{2}$ for all sufficiently large $n \in \mathcal{N}$, and hence for such $n$, (4.2) holds with, e.g., $C^{\prime}=7+32 C$. This concludes the proof.

It is well-known that, when $p_{n}=1 / 2$, the so-called Tribes function (see, e.g., Section 4.2 in [14]) is increasing and transitive and satisfies $I\left(f_{n}\right)=C \log n$. Moreover, a sequence of Tribes functions is noise sensitive, and hence non-tame by Proposition 1.17 in [10]. In particular, this shows that the inequality in Lemma 4.1 does not hold for all non-tame sequences of increasing and transitive Boolean functions. The main idea in the proof of Theorem 1.4 will instead be to show that (4.1) holds for all sequences of regular Boolean functions which are not noise sensitive. This is the main motivation for the next lemma.

Lemma 4.3. Let $\left(f_{n}\right)_{n \in \mathcal{N}}$ be a non-degenerate sequence of regular and increasing Boolean functions. Assume further that $p_{n} \leqslant 1 / 2$ and $\liminf _{n \rightarrow \infty} n p_{n}=\infty$. Then either $\left(f_{n}\right)_{n \in \mathcal{N}}$ is not tame, or

$$
\lim _{n \rightarrow \infty} p_{n}^{-1} \sum_{i=1}^{n} I_{i}\left(f_{n}\right)^{2}=0
$$

Proof of Lemma 4.3. Assume that $\limsup _{n \rightarrow \infty} p_{n}^{-1} \sum_{i \in[n]} I_{i}\left(f_{n}\right)^{2}>0$. We need to show that in this case, $\left(f_{n}\right)_{n \geqslant 1}$ is not tame. To this end, note that when

$$
\limsup _{n \rightarrow \infty} p_{n}^{-1} \sum_{i \in[n]} I_{i}\left(f_{n}\right)^{2}>0
$$


When are sequences of Boolean functions tame?

there is a constant $D>0$ and an infinite increasing subsequence $\mathcal{N}^{\prime}=\left(n_{1}, n_{2}, \ldots\right)$ of $\mathcal{N}$ such that $\sum_{i \in[n]} I_{i}\left(f_{n}\right)^{2}>D p_{n}$ for all $n \in \mathcal{N}^{\prime}$. Since $f_{n}$ is regular for all $n \in \mathcal{N}$, and $\mathcal{N}^{\prime}$ is a subsequence of $\mathcal{N}$, it follows that, for all $n \in \mathcal{N}^{\prime}$, we have

$$
I\left(f_{n}\right)^{2}=\left(n I_{1}\left(f_{n}\right)\right)^{2}=n \cdot n I_{1}\left(f_{n}\right)^{2}=n \cdot \sum_{i \in[n]} I_{i}\left(f_{n}\right)^{2} \geqslant n \cdot D p_{n}=D \cdot p_{n} n .
$$

Since $\operatorname{Var}\left(f_{n}\right)\left(1-p_{n}\right)<1$ for all $n \in \mathcal{N}$, it follows that

$$
p_{n}\left(1-p_{n}\right) n \operatorname{Var}\left(f_{n}\right) \leqslant D^{-1} I\left(f_{n}\right)^{2} .
$$

On the other hand, since, by assumption, $\lim _{n \rightarrow \infty} n p_{n}=\infty, p_{n} \leqslant 1 / 2$, and $\left(f_{n}\right)_{n \in \mathcal{N}}$ is non-degenerate, it follows from the previous equation to together with the observation that $\mathbb{E}\left[C_{f}\right]=I\left(f_{n}\right)$ that $\lim _{n \rightarrow \infty} \mathbb{E}\left[C_{f}\right]=\infty$. Consequently, we can apply Lemma 4.1 to deduce that $\left(f_{n}\right)_{n \in \mathcal{N}^{\prime}}$ is not tame. Since $\mathcal{N}^{\prime}$ is a subsequence of $\mathcal{N}$, it follows that $\left(f_{n}\right)_{n \in \mathcal{N}}$ is not tame. This concludes the proof.

Proof of Theorem 1.4. Let $\left(f_{n}\right)_{n \in \mathcal{N}}$ be a non-degenerate sequence of regular and increasing Boolean functions, and assume that $0<\liminf _{n \rightarrow \infty} p_{n} \leqslant \limsup _{n \rightarrow \infty}<1$. Note that if $\left(f_{n}\right)_{n \in \mathcal{N}}$ is noise sensitive then, by Proposition 1.17 in [10], $\left(f_{n}\right)_{n \in \mathcal{N}}$ is volatile and hence not tame. Consequently, we can assume that $\left(f_{n}\right)_{n \in \mathcal{N}}$ is not noise sensitive. Since $0<\liminf _{n \rightarrow \infty} p_{n} \leqslant \limsup _{n \rightarrow \infty} p_{n}<1$, it follows from Theorem 7 in [11] that $\limsup _{n \rightarrow \infty} \sum_{i \in[n]} I_{i}\left(f_{n}\right)^{2}>0$. The desired conclusion thus follows from applying Lemma 4.3 .

Remark 4.4. The proof of Theorem 1.4 does not work when $\lim _{n \rightarrow \infty} p_{n}=0$, even though Theorem 7 in [11], as well as the similar Theorem 1.3 in [3], holds also in this case. The reason for this is that when $\lim _{n \rightarrow \infty} p_{n}=0$, the lower bound on $p_{n}^{-1} \sum_{i \in[n]} I_{i}\left(f_{n}\right)^{2}$ provided by these theorems are too weak to be used in conjunction with Lemma 4.3. Theorem I.5 in [12] gives an alternative to Theorem 7 in [11] for sequences of increasing Boolean functions when $\left(p_{n}\right)_{n \geqslant 1}$ is given by $p_{n}=n^{-(k-1) / k}$ for some even number $k \geqslant 0$. However, this theorem has additional assumptions which, e.g., the counter-example given in [6] does not satisfy. More important for us however, it does not cover the range of $\left(p_{n}\right)_{n \geqslant 1}$ where we have neither counter-examples nor a positive result.

\section{References}

[1] Benjamini, I., Kalai, G., Schramm, O.: Noise sensitivity of Boolean functions and applications to percolation. Publ. Math. Inst. Hautes Études Sci. 90, (1999), 5-43. MR1813223

[2] Bourgain, J., Kahn, J., Kalai, G., Katznelson, Y. Linial, N.: The influence of variables in product spaces. Israel J. of Math. 77 (1), (1992), 55-64. MR1194785

[3] Bouyrie, R.: On quantitative noise stability and influences for discrete and continuous Models. Combin. Probab. Comput. 27 (3), (2028), 334-357. MR3788164

[4] Eldan, R., Gross, R.: Concentration on the Boolean hypercube via pathwise stochastic analysis. STOC 2020: Proceedings of the 52nd Annual ACM SIGACT Symposium on Theory of Computing, (2020), 208-221. MR4141754

[5] Forsström, M. P.: Denseness of volatile and nonvolatile sequences of functions. Stochastic Process. Appl. 128 (11), (2018), 3880-3896. MR3860013

[6] Forsström, M. P.: A tame sequence of transitive Boolean functions. Electron. Commun. in Probab. 25 (83), (2020), 1-8. MR4195177

[7] Friedgut, E., Kalai, G.: Every monotone graph property has a sharp threshold. Proc. Amer. Math. Soc. 124 (10), (1996). MR1371123

[8] Galicza, Pal.: Pivotality versus noise stability for monotone transitive functions. Electron. Commun. Probab. 25, (2020). MR4069737 
When are sequences of Boolean functions tame?

[9] Garban, C., Steif, J. E.: Noise sensitivity of Boolean functions and percolation. Cambridge Univ. Press, (2014). MR3468568

[10] Jonasson, J., Steif, J. E.: Volatility of Boolean functions, Stochastic Process. Appl., 126 (10), (2006), 2956-2975. MR3542622

[11] Keller, N., Kindler, G.: Quantitative Relation Between Noise Sensitivity and Influences. Combinatorica 33, (2013), 45-71. MR3070086

[12] Lifshitz, N., Minzer, D.: Noise Sensitivity on the p-Biased Hypercube. 2019 IEEE 60th Annual Symposium on Foundations of Computer Science (FOCS), (2019). MR4228222

[13] Mossel, E., O’Donnell, R., Oleszkiewicz, K.: Noise stability of functions with low influences: Invariance and optimality. Ann. of Math. 171 (1), (2010), 295-341. MR2630040

[14] O'Donnell, R.: Analysis of Boolean functions. Cambridge Univ. Press, (2014). MR3443800

[15] O’Donnell, R., Saks, M., Schramm, O., Servedio, R. A.: Every decision tree has an influential variable. 46th Annual IEEE Symposium on Foundations of Computer Science, (2005). arXiv:cs/0508071

[16] Talagrand, M.: How much are increasing sets positively correlated? Combinatorica 16 (2), (1996), 243-258. MR1401897

Acknowledgments. The author would like to thank Gil Kalai and Jeffrey E. Steif for comments on the contents of this paper. Also, the author is grateful to an anonymous referee for the many useful comments, including suggesting several improvements of the proofs in this paper, especially to the proof of Lemma 3.2, and also for pointing out the relationship to the Majority function, now mentioned in Remark 4.2. Finally, the author is grateful to an anonymous referee on the companion paper [6], for making several interesting comments of relevance for this paper. 


\section{Electronic Journal of Probability Electronic Communications in Probability}

\section{Advantages of publishing in EJP-ECP}

- Very high standards

- Free for authors, free for readers

- Quick publication (no backlog)

- Secure publication $\left(\mathrm{LOCKSS}^{1}\right)$

- Easy interface (EJMS²)

\section{Economical model of EJP-ECP}

- Non profit, sponsored by $\mathrm{IMS}^{3}, \mathrm{BS}^{4}$, ProjectEuclid ${ }^{5}$

- Purely electronic

\section{Help keep the journal free and vigorous}

- Donate to the IMS open access fund ${ }^{6}$ (click here to donate!)

- Submit your best articles to EJP-ECP

- Choose EJP-ECP over for-profit journals

\footnotetext{
${ }^{1}$ LOCKSS: Lots of Copies Keep Stuff Safe http://www. lockss.org/

${ }^{2}$ EJMS: Electronic Journal Management System http://www.vtex.lt/en/ejms.html

${ }^{3}$ IMS: Institute of Mathematical Statistics http://www.imstat.org/

${ }^{4}$ BS: Bernoulli Society http://www. bernoulli-society.org/

${ }^{5}$ Project Euclid: https://projecteuclid.org/

${ }^{6}$ IMS Open Access Fund: http://www.imstat.org/publications/open.htm
} 